\section{Research Square}

Preprints are preliminary reports that have not undergone peer review.

They should not be considered conclusive, used to inform clinical practice, or referenced by the media as validated information.

\title{
Distinct CD8 T cell populations with differential exhaustion profiles associate with secondary complications in common variable immunodeficiency
}

Adam Klocperk ( $\nabla$ adam.klocperk@fnmotol.cz )

University Hospital Motol: Fakultni nemocnice v Motole https://orcid.org/0000-0002-1526-4557

David Friedmann

Alexandra Emilia Schlaak

Susanne Unger

Zuzana Parackova

Sigune Goldacker

Anna Sediva

Bertram Bengsch

Klaus Wamatz

\section{Research Article}

Keywords: Immunodeficiency, T cells, CVID, exhaustion, activation, differentiation

Posted Date: May 3rd, 2022

DOI: https://doi.org/10.21203/rs.3.rs-1359086/v2

License: @ (i) This work is licensed under a Creative Commons Attribution 4.0 International License. Read Full License 


\section{Abstract}

Purpose Common variable immunodeficiency (CVID) is the most frequent symptomatic primary immunodeficiency, with heterogeneous clinical presentation. Our goal was to analyze CD8 T cell homeostasis in patients with infection only CVID, compared to those additionally affected by dysregulatory and autoimmune phenomena. Methods Flow and mass cytometry evaluation of peripheral blood of 40 patients with CVID and 17 healthy donors. Results CD8 T cells are skewed in patients with CVID, with loss of naïve and increase of effector memory stages, expansion of cell clusters with high functional exhaustion scores, and a highly activated population of cells with immunoregulatory features, producing IL-10. These findings correlate to clinically widely used B-cellbased EURO classification. Features of exhaustion, including loss of CD127, CD28 and expression of TIGIT and PD-1 in CD8 T cells are strongly associated with interstitial lung disease and autoimmune cytopenias, whereas CD8 T cell activation with elevated HLA-DR and CD38 expression predict non-infectious diarrhea. Conclusion We demonstrate features of advanced differentiation, exhaustion, activation and immunoregulatory capabilities within CD8 T cells of CVID patients. Assessment of CD8 T cell phenotype may allow risk-assessment of CVID patients, and provide new insights into CVID pathogenesis, including a better understanding of mechanisms underlying $T$ cell exhaustion and regulation.

\section{Introduction}

Common variable immunodeficiency (CVID), the most prevalent symptomatic primary immunodeficiency, is characterized by decreased production of immunoglobulins and impaired ability of B cells to produce high affinity antigen-specific lgG antibodies after vaccination [1]. The disease is clinically highly heterogeneous, and two subgroups of patients are commonly recognized on clinical basis, those who only suffer from increased susceptibility to infections, and those with a more complex disease featuring additionally phenomena of immune dysregulation, including autoimmune hemolytic anemia, interstitial lung disease (ILD), persistent non-infectious diarrhea and others [2].

The humoral defect in CVID is associated with an altered B cell homeostasis and the presence or absence of B cell subpopulations has been widely used for disease classification [3].

CD4 T-cell studies have shown a reduction of recent thymic emigrant and naïve CD4 T cells $[4,5]$ and an early commitment to the follicular lineage in patients with autoimmune cytopenias [6] possibly driven by bacterial endotoxemia, which concurrently impairs CD4 T-cell proliferative capacity and production of IFN$Y$ and IL-2 [7]. Reinforcing the notion that the cellular immunity is altered in patients with complicated form of CVID, our group previously noted a shift towards IFN-y-producing Th1 memory and follicular helper T cells [8], a finding which extended from peripheral blood into tissues like the lung and broncho-alveolar fluid of CVID patients with ILD [9] and the gastrointestinal tract [10]. A recent study has also shown higher expression of inhibitory receptors PD-1, LAG3, CTLA4 and TIGIT in memory CD4 T cells of patients with complex CVID, which nevertheless retained capacity to produce IFN-y and proliferate [11].

The CD8 compartment in CVID remains less explored, with studies reporting normal [4,12], decreased [13] or increased [14] absolute CD8 T-cell counts, but unanimously describing higher proportion of overall CD8 T cells, with low proportions of naïve, but elevated effector memory CD8 T cells [15,16] and terminal effector cells expressing CD57 and KLRG1 [17], altered response to TLR stimulation [18], substantial activation measured by the expression of HLA-DR and CD38 [19-22], and increased expression of cytotoxic markers such as granzyme B [19] in patients with autoimmune complications and lymphoproliferation. CD8 T cells are involved in the pathogenesis of some manifestations such as enteropathy [23], hepatopathy [24] and potentially even in ITP [25]. Prolonged stimulation by both autoantigens and external pathogens may ultimately result in chronic activation and ongoing inflammation, accelerating CD $8 \mathrm{~T}$ cell differentiation and possibly leading to features of CD8 T-cell exhaustion described in detail in other diseases with chronic inflammation [26,27]. However, the role of T cell exhaustion in CVID remains unclear.

Thus, we set out to utilize recent advances in understanding of CD8 T cell biology, especially with focus on their differentiation, exhaustion and progenitor capacity, using highly multiplexed immune phenotyping and unsupervised exploratory approaches in a large cohort of genetically diverse patients with infection only and complicated CVID. A challenge in profiling T-cell exhaustion is the overlap of exhaustion markers with effector or memory T cell populations. We therefore used a multifaceted exhaustion profiling approach that focuses on analysis of markers highly biased to expression in exhausted $T$ cells in combination with a high-dimensional single-cell phenotyping approach as well as functional assays to profile cytokine co-expression patterns typical for exhausted T cells $[28,29]$. Our work points to the expansion of exhausted CD8 T cells with immunoregulatory features particularly in CVID patients with complex disease manifestation, suggesting them as possible contributors to disease activity but also highlighting them as potential biomarkers for high-risk patients.

\section{Methods \\ Patients and samples}

Peripheral blood was obtained from patients with CVID seen at the immunodeficiency clinic of the Freiburg University Medical Center, Freiburg, Germany. All patients fulfilled the criteria for CVID according to the European Society for Immunodeficiencies (www.esid.org). The following clinical data were recorded: splenomegaly (defined as a diameter of $>11 \times 4 \times 7 \mathrm{~cm}$, as shown using ultrasonography or computed tomography [CT]), generalized lymphadenopathy (lymph nodes $>1 \mathrm{~cm}$ in diameter in >= 2 different anatomic sites detected by means of clinical examination, ultrasonography, or CT), granulomatous disease (suggested by CT or proven by histology), autoimmune cytopenias (autoimmune hemolytic anemia or thrombocytopenia), interstitial lung disease (based on CT morphology and bronchoalveolar lavage or biopsy), hepatopathy (based on ultrasound, elastography, duplex, laboratory parameters), and enteropathy (exclusion of infection and histology). Patients with only splenomegaly or lymphadenopathy were classified as "infection only" (CVIDio), whereas patients with any of the other complications were classified as "complex disease" (CVIDc). Patients who never underwent the respective diagnostic workup were 
excluded from the analysis. Patients were further classified according to EUROclass classification, based on the reduction of switched memory B (smB) cells and the expansion of CD21low B cells. Description of patient cohort can be inspected in Table 1.

\section{Flow cytometry}

Following peripheral venipuncture, peripheral blood mononuclear cells (PBMCs) were isolated from ethylenediaminetetraacetic acid (EDTA)-anticoagulated blood by centrifugation on a density gradient Pancoll (PAN Biotech, Aidenbach, Germany), then frozen at $-180^{\circ} \mathrm{C}$ in $10 \%$ dimethyl sulfoxide (DMSO) and $20 \%$ fetal bovine serum (FBS). Samples were thawed in batches, washed 2 times with RPMI 1640 (Pan Biotec, Aidenbach) + 10\% FCS (Merck, Darmstadt, Germany) $+1 \%$ PenStrep (Life Technologies, Darmstadt, Germany), and labeled with respective antibodies for detection of indicated cell-surface molecules. When applicable, fixation and permeabilization was performed with the eBioscience Intracellular Fixation \& Permeabilization Buffer Set (ThermoFisher Scientific, Darmstadt, Germany). Data was acquired on LSR II flow cytometer (BD Biosciences, Franklin Lakes, NJ) and analyzed with FlowJo software (version 7.6.5 or 10.5.3, TreeStar, Ashland, Ore). List of used antibodies can be viewed in Table S1. The gating strategy is shown in Figure S1.

\section{Mass cytometry}

For mass cytometry, 2 million viable PBMCs per patient (4 HD, 4 CVIDio, 4 CVIDc) were stimulated with 0.05 $\mu \mathrm{g} / \mathrm{ml}$ phorbol 12-myristate 13-acetate (PMA) and $1 \mu \mathrm{g} / \mathrm{ml}$ ionomycin for 5 hours, with $0.5 \mu \mathrm{g} / \mathrm{ml}$ GolgiPlug and $0.032 \mu \mathrm{g} / \mathrm{ml}$ GolgiStop added 1 hour into the stimulation. Cells were then stained for mass cytometry. Mass cytometry reagents were obtained or generated by custom conjugation to isotope-loaded polymers using the Maxpar X8 antibody labeling kit. Mass cytometry antibodies used are shown in Table S2. Staining was performed after $\beta 2$-microglobulin-based sample barcoding. Briefly, single-cell suspensions were pelleted, incubated with 20mM Lanthanum-139-loaded maleimido-mono-amine-DOTA in PBS for $5 \mathrm{~min}$ at RT for live/dead discrimination (LD). Cells were washed in staining buffer and resuspended in surface antibody cocktail, incubated for 30min at RT, washed twice in staining buffer, fixed and permeabilized using FoxP3 staining buffer set (ThermoFisher Scientific), and stained intracellularly for 60min at RT. Cells were further washed twice before fixation in 4\% PFA solution containing 125nM Iridium overnight at 4C. Prior to data acquisition on CyTOF Helios (Fluidigm), cells were washed twice in PBS and once in cell acquisition solution. After acquisition, all CyTOF files were normalized together using the Bead-based Nolan Lab normalizer (available from https://github.com/nolanlab/bead-normalization/releases). Afterwards, clean-up of the FCS files was performed with FlowJoV10 by using Gaussian-derived parameters "Residual", "Center", "Offset", "Width", "Event Length", MM-Dota and Iridium to eliminate dead cells, debris and normalization beads. Files were concatenated using FlowJoV10 prior to manual debarcoding. For data analysis, including Wanderlust [30], FlowSOM [31] and UMAP [32], the online data analysis platform OMIQ (www.omiq.ai) was used (Omiq, Santa Clara, CA). For Wanderlust, FlowSOM and UMAP, pseudotime, clustering and dimensionality reduction was calculated based on the following markers: 2B4, CCR7, CD7, CD8, CD16, CD27, CD28, CD38, CD39, CD45RA, CD57, CD73, CD127, CD120b, CD160, CTLA-4, CXCR5, Eomes, FOX01, FoxP3, Helios, HLA-DR, LAG-3, PD-1, Tbet, TCF1, TIGIT, Tim3 and TOX. A functional exhaustion score (FES) was calculated using the production of IL-2 and XCL-1, as well as IFN- $\gamma$ and TNF- $a$ coproduction $\left(2 *\left(\% I F N-\gamma^{+} T N F-\alpha^{-}\right)-\left(\% \text { IFN- } \gamma^{-} \text {TNF- } a^{+}\right)-\left(\% \text { IL-2 } 2^{+}\right)\right)^{*}\left(\% X C L-1^{+}\right)[28]$.

\section{Study approval}

Written informed consent with participation in this study was obtained from all patients and control subjects in accordance with the ethical standards of the institutional and national research committee and with the 1964 Helsinki Declaration and its later amendments. The study was approved by local authorities (University of Freiburg Ethics Committee 239/1999 and 121/11).

\section{Statistical analysis}

In the boxplots used throughout the manuscript, boxes depict the 25th and 75th percentiles (first and third quartile, respectively) and whiskers depict the 2.597.5th percentiles. In violin plots, medium smoothing was used and the plots span from lowest to highest measurement, with median, $1^{\text {st }}$ and $3^{\text {rd }}$ quartiles shown. Tests used are specified in each results section.

Statistical analyses and the generation of graphs were performed in the statistical language and environment R, version 3.6.3, GraphPad Prism software (version 8.0.1, GraphPad Software, San Diego, CA, USA), and Microsoft Excel 2016 (Microsoft, Redmond, WA, USA). The Simplified Presentation of Incredibly Complex Evaluations (SPICE) plots shown in Figure 1 were constructed using the SPICE 6 software [33].

\section{Results}

\section{Relative expansion of CD8 T cells in CVID comprises mainly increased percentages of EM2 and EM3 subpopulations}

30 adult patients with CVIDc (17 female, 13 male), 10 adult patients with CVIDio ( 3 female, 7 male) and 17 healthy adults with no history of immune disease were included in this study. Detailed description of the cohort, including genetic findings, Freiburg and EUROclass classifications, can be found in Table 1. The most common clinical complications were recurrent non-infectious diarrhea $(n=21)$, interstitial lung disease (ILD, $n=17)$ and lymphadenopathy $(L A D, n=16)$, followed by autoimmune cytopenia (AIC, $n=14$ ). Splenomegaly was present in 31 patients, but when found in isolation with no other clinical complications did not qualify the patient as CVIDc. A graph showing co-incidence of various clinical features in the studied cohort can be found in Figure $1 \mathrm{~A}$, and the proportion of patients in the different EUROclass phenotypes can be found in Figure 1B.

Page $3 / 13$ 
We next performed a detailed analysis of the differentiation and exhaustion of CD8 T cells in CVID patients. Patients with CVID had higher proportion of CD8 T cells than healthy controls $(\mathrm{HD})$ in the peripheral blood (Figure $1 \mathrm{C}$ ) (Welch's ANOVA $p=0.008$, unpaired t-tests with Welch's correction) (CD4:8 ratio in Figure S2), with a shift from naïve (CD45RA $\left.{ }^{+} C C R 7^{+}\right)$towards effector memory (EM, CD45RA-CCR7) and terminal effector memory cells (TEMRA, CD45RA ${ }^{+}$CCR7 $)$ stages (Figure 1D). In particular, EM2 (CD27 $\left.{ }^{+} 8^{-}\right)$and EM3 (CD27-28) cells were significantly expanded in both CVIDio and CVIDc compared to HD (Figure 1E) (CVIDio EM2 unpaired t-test with Welch's correction $p<0.001$, EM3 $p=0.007$, CVIDc EM2 $p=0.002$, EM3 $p=0.01$ ).

For detailed exhaustion profiling, where phenotyping of T cells based on differentiation markers is insufficient to precisely identify exhausted T-cell populations, we combined a highly multiplexed phenotyping of exhaustion-biased immune markers with a functional analysis of the expression patterns of ( $\mathrm{n}$ $=10$ ) cytokines important for exhausted T-cell biology. This advanced approach allows for the identification of relevant exhausted T-cell phenotypes and evaluation of an exhaustion-typical dysfunction pattern (i.e. reduced IL-2 and TNF-a production, but possible production of IFN- $y$ and XCL-1 chemokine) that can be assessed on a single-cell level using a functional exhaustion score, as previously published [28]. For this reason, we stimulated PBMCs from 4 healthy donors, 4 CVIDio (patient ID 31, 33, 38, 40) and 4 CVIDc (patient ID 5, 10, 17, 23) patients with PMA + ionomycin and analyzed them by using a mass cytometry panel of 44 extracellular markers, transcription factors, cytokines and chemokines (for a complete list of markers see Table S2).

To assess if the analysis of the pre-defined canonical T cell subsets correlated with data-driven approaches, we then performed trajectory-inference analysis using Wanderlust [30] based on 29 phenotypic markers (for a list see Methods). The resulting pseudotime variable corresponded well with these canonical differentiation stages from naïve into central, effector memory and ultimately TEMRA populations (Figure 1F, G), documenting expected changes with gradual decrease and final re-expression of CD45RA, loss of CCR7, preserved in CM, and loss of CD27 and CD28.

When stimulated with PMA and ionomycin, the EM2 and EM3 CD8 T cells had a unique phenotype which on average had decreased production of IL-2, but high production of cytotoxic molecules perforin, granulysin, and the lymphotactin XCL-1 (Figure 1H, dotplots in Figure S3). Especially EM3 cells also displayed a high expression of the senescence marker CD57, increased in chronic immune activation [34], the exhaustion marker Tim3, the ectonucleotidase CD39 and the TNF-a receptor CD120b. Together, this data revealed significant heterogeneity of exhaustion markers and functional T cell profiles in canonical T cell differentiation subsets.

\section{CD8 T cell landscape is grossly disturbed in CVID, with enriched activated and exhausted cellular populations and cells with immunoregulatory potential, but lacking progenitor capability}

To understand the exhaustion programs of CD8 T cells in CVID in more detail, we analyzed the high-dimensional dataset in a data-driven, unbiased manner. Dimensionality reduction on 29 phenotypic markers was performed by UMAP, revealing major differences between HD, CVIDio and CVIDc cohorts (Figure 2A). Clustering of high-dimensional data by a self-Organizing Maps algorithm (FlowSOM) revealed 20 distinct cell clusters (optimal number of clusters selected using the elbow method) (Figure 2B), the proportion of which was significantly different between the three cohorts (Figure 2C, Figure S4). Cluster 8 was significantly expanded in CVIDc compared to HD (unpaired t-test with Welch's correction $p=0.0002$ ), cluster 16 was significantly expanded in CVIDio compared to HD ( $p=0.0002, C V I D c \times H D p=0.1)$, whereas cluster 18 was significantly diminished in both CVIDio and CVIDc compared to HD (CVIDio $\times$ HD $p$ $=0.01, C V I D c \times H D p=0.009$ ). Several other clusters (cluster $3,6,10,12$ ) were insignificantly expanded in both CVID cohorts. The clusters provided higher granularity compared to the canonical differentiation stages shown in Figure 1 and showed different localization across the canonical developmental pseudotime (Figure 2E).

We then compared the expression of all phenotypic and functional markers across all 20 clusters (Figure 2D). Further, we calculated a functional exhaustion score (FES) as described previously to identify clusters with a polyfunctionality pattern typical for exhausted T cells [28], based on TNF-a, IFN- $\gamma$, IL-2 and XCL-1 production. FES was mediocre or high in clusters expanded in CVIDc, with a similar trend in CVIDio, and low in clusters more prevalent in healthy donors, and it was significantly different between clusters (Welch's ANOVA $p<0.0001$, Figure S5). These findings link clusters with an exhaustion phenotype and FES to CVID, in particular in CVIDc patients.

Clusters 3 and 16, which were upregulated in CVID, displayed strong expression of the activation markers CD38, HLA-DR, the proliferation-associated nuclear protein Ki67, and produced IL-10, IL-17A, to a lesser extent also the effector cytokine IFN- $\gamma$, but little or none TNF-a as well as no perforin and granulysin. Their expression patterns then diverged, with cluster 3 having low expression of most exhaustion-associated markers, whereas cluster 16 displayed an elevated proportion of cells staining positive for CXCR5, higher expression levels of transcription factors TOX, FOXO1, Helios and Eomes, and inhibitory molecules such as PD-1, TIGIT, LAG3, Tim3 and CTLA-4, and had a particularly high FES. The phenotype of cells in cluster 16 is reminiscent of the elusive regulatory CD8 T cell population, based on their production of IL-10, PD-1 expression and higher average expression of the transcription factor FoxP3 in comparison to other CD8 T cells (Figure S6) [35,36].

Cluster 6 bore the hallmarks of senescent cells expressing CD57 and effector capabilities.

Cluster 8, also expanded in CVIDc, displayed an above average expression of exhaustion-associated phenotypic markers Eomes, PD-1, TIGIT, LAG-3, 2B4 and CD160, with low Tbet and TCF1, suggesting terminal differentiation. These cells retained their capacity to produce TNF-a, IFN- $\gamma$ and XCL-1, but did not produce large amounts of cytotoxic molecules such as granulysin or perforin and unlike cluster 16 were not activated or proliferating. These cells together with cluster 12 , which had very high expression of PD-1, fulfil most canonical signs of being exhausted and have a correspondingly high FES.

Cluster 18, severely diminished in CVID, had the hallmarks of naïve CD8 T cells, including expression of CD45RA, CCR7, CD27, CD28, CD73, CD127 and CD7. 
Cluster 20, a small population but seemingly lower in CVID compared to HD, clustered together with the naïve CD8 T cells of cluster 18, but was notable for its high expression of TCF1 and FOX01, transcription factors associated with progenitor capabilities of effector cells, in conjunction with CCR7. Similarly, cluster 19 also expressed TCF1, but also high levels of CXCR5 intermediate levels of PD-1 and no TOX, which bears similarity to progenitor population of exhausted T cells $[37,38]$. Cluster 19 was comparably prevalent in both CVID patients and HD.

Thus, the CyTOF analysis revealed the reduction of naïve CD8 T cells and CCR7 ${ }^{+} \mathrm{TCF} 1^{+} \mathrm{FOXO} 1^{+}$stem-cell-like progenitors, but not exhaustion-specific CXCR5 ${ }^{+} \mathrm{PD}-1^{\text {int }} \mathrm{TCF} 1^{+}$progenitors. In contrary, two distinct groups of cells were more prevalent in CVID, a group of highly differentiated classical exhausted cells with high FES lacking signs of ongoing activation and production of cytokines, and a group of highly activated and proliferating cells with immunoregulatory features such as the production of IL-10 and expression of CXCR5 and higher average expression of FoxP3.

\section{Bulk CVID CD8 T cells show features of exhaustion, activation and cytotoxicity}

Following the markers of interest discovered by unbiased clustering approach shown above in a smaller cohort assessed by mass cytometry, we verified the expression pattern of exhaustion-associated markers PD-1, TIGIT, CD127, 2B4, Tbet, Eomes and TCF1, activation markers CD38 and HLA-DR, nuclear protein Ki67 and the cytotoxicity markers perforin and granzyme B across several flow cytometric panels in the larger cohort of 40 patients and 17 healthy donors (Figure $3 \mathrm{~A}$ and S7).

On average, bulk CVIDc derived CD8 T cells were activated (Figure 3B), proliferating (Figure 3C) and functionally active by producing cytotoxic molecules (Figure 3D), corresponding to the expanded clusters 3, 8, 12 and 16. These features were generally more pronounced in CVIDc derived CD8 T cells compared to CVIDio, although the difference only reached significance for the percentage of activated HLA-DR ${ }^{+}$cells. CVID CD8 T cells on average had higher expression of the exhaustion-associated marker TIGIT and lost the expression of the IL-7 receptor CD127, while the proportion of PD- $1^{+}$cells was comparable between HD, CVIDio and CVIDc (Figure 3E). These changes did not merely reflect changes in the distribution of the different memory stages shown in Figure 1, but were also present within the distinct naïve and EM subpopulations (Figure S8).

To assess cells corresponding to the dysregulated clusters seen in CyTOF analysis using the larger cohort of 40 patients and 17 healthy donors stained with flow cytometry, we gated on CD127-PD- $1^{+} \mathrm{TIGIT}^{+} \mathrm{CD} 28^{-}$cells (phenotype equivalent of clusters 8 and 12 , hereafter referred to also as Cluster $8 / 12$-like cells) and CD127-PD- high $^{\text {TIGIT }}{ }^{+}$CD $28^{+}$cells (phenotype equivalent of cluster 16 , hereafter referred to also as Cluster 16 -like cells). Cluster $8 / 12$-like cells were significantly more prevalent in CVIDc compared to HD, with a similar but insignificant trend for cluster 16-like cells (Figure 3F). The prevalence of cluster $8 / 12$ like and cluster 16 -like cells determined by flow cytometry correlated to CyTOF-determined clusters $8+12$ and cluster 16 cells in those patients assessed by both methods $(n=12)$ (Figure 3G) (Pearson correlation $p=0.04$ and $0.0024, R^{2}=0.354$ and 0.618 , respectively).

Retrospectively re-assessing the CyTOF data of 4 HD, 4 CVIDio and 4 CVIDc patients, we verified that the expression pattern of CD127-PD-1 ${ }^{+}$TIGIT $^{+} \mathrm{CD}^{-2} 8^{-}$cells mirrored that of clusters 8 and 12, the expression pattern of CD127-PD-1 high TIGIT $^{+}$CD28 ${ }^{+}$mirrored that of cluster 16 (Figure $3 \mathrm{H}$ ), and when overlaid on the UMAP visualization these populations overlapped with their respective clusters (Figure 3I). Functionally, CD $127^{-}$PD $-1^{\text {high }}{ }^{-} I_{G I T}{ }^{+}$CD $28^{+}$expressed high levels of Ki67, IL-10 and IL-2, but unlike their CD127-PD- $1^{+} \mathrm{TIGIT}^{+} \mathrm{CD} 28^{-}$counterparts lacked the cytotoxic effectors perforin and granulysin.

The expansion of $\mathrm{TIGIT}^{+} \mathrm{CD} 8 \mathrm{~T}$ cells was strongly associated with the EURO classification based on B cell phenotyping (see Methods and [3]) (one-way ANOVA $p=0.002$ ) (Figure $3 \mathrm{~J}$ ), as was the expression of PD-1 (one-way ANOVA $p=0.035$ ) and crucially so were the exhausted cluster 8/12-like cells (CD127 $\mathrm{PD}-1^{\text {int }} \mathrm{TIGIT}^{+} \mathrm{CD} 28^{-}$) (one-way ANOVA $\mathrm{p}=0.0429$ ). The association between the EURO classification and immunoregulatory cluster 16 -like cells (CD127-PD$1^{\text {high }} \mathrm{TIGIT}^{+} \mathrm{CD} 28^{+}$) did not reach significance (one-way ANOVA $\mathrm{p}=0.58$ ) (Figure $3 \mathrm{~K}$ ).

These results show activation and elevated expression of exhaustion-associated molecules on bulk CD8 T cells in CVID patients. Further, they demonstrate a surrogate gating strategy to assess exhausted cells corresponding to clusters 8 and 12 and immunoregulatory cells corresponding to cluster 16 using lowparametric flow cytometry and show that these exhausted cells and markers are associated with the EURO classification system of CVID.

\section{Clinical complications of CVID such as interstitial lung disease and autoimmune cytopenia strongly associate with more pronounced features of exhaustion, whereas diarrhea associates with $\mathrm{T}$ cell activation}

As the clinical phenotype of CVIDc is varied and patients can exhibit many different complications, we tested the association between CD8 T cell phenotype and individual clinical features seen in patients with complex disease. Figure 4A shows a table with unpaired student's t-test with Welch's correction nonadjusted p-values calculated between all CVID patients with and without each symptom for select easy-to-measure parameters, exhausted cluster $8 / 12$-like and immunoregulatory cluster 16-like cells. Whereas the expression of inhibitory receptors, loss of CD127 and the exhausted cluster 8/12-like cells were significantly associated with AIC, lymphadenopathy and ILD, recurrent non-infectious diarrhea was most strongly associated with CD8 T-cell activation and the activated immunoregulatory cluster 16 -like cells.

ILD was strongly associated with the proportion of cluster 8/12-like cells, loss of CD127 and expression of TIGIT (Figure 4B). Cluster 8/12-like (CD127-PD$\left.1^{+} \mathrm{TIGIT}^{+} \mathrm{CD} 28^{-}\right)$cells offered best combination of sensitivity and specificity when searching for ILD in CVID patients $(p=0.0057$, area under the receiver operating characteristic (ROC) curve (AUC) $=0.791,>12 \%$ cluster $8 / 12$-like cells had $77 \%$ sensitivity and $74 \%$ specificity for ILD), however TIGIT ${ }^{+}$cells also performed very well $\left(p=0.0123\right.$, AUC $=0.734,>66 \%$ TIGIT $^{+}$cells had $64 \%$ sensitivity and $74 \%$ specificity for ILD) (Figure 4F).

Page 5/13 
AIC was also associated with loss of CD127 and expression of TIGIT (Figure 4C). While TIGIT $^{+}$cells were significant predictors of AIC $(p=0.014, A U C=0.736$, $>68 \%$ TIGIT $^{+}$cells had $64 \%$ sensitivity and $81 \%$ specificity for AIC), TIGIT/CD127 ratio was even more helpful $(p=0.009$, AUC $=0.751,>1.5$ ratio of $\mathrm{TIGIT}^{+} / \mathrm{CD} 127^{+}$cells had $64 \%$ sensitivity and $81 \%$ specificity for AIC).

Diarrhea in CVID was not associated with an expansion of the regulatory cluster 16-like cells as it was seen in patients without diarrhea when compared to healthy donors (Figure 4D). Instead, the expression of activation markers CD38 ( $p=0.002, A U C=0.784,>41 \%$ CD $38^{+}$cells had $71 \%$ sensitivity and $84 \%$ specificity for diarrhea) and HLA-DR ( $p=0.006$, AUC $=0.753,>6 \% \mathrm{HLA}^{-D R^{+}}$cells had $71 \%$ sensitivity and $63 \%$ specificity for diarrhea) were both good predictors of this complication.

A similar distribution of cluster 16-like cells and additionally bulk CD8 T cells was seen for the manifestation of hepatopathy (Figure 1E). The best predictor for hepatopathy in CVID patients was the low proportion of EM1 cells $(p=0.028$, AUC $=0.763,<9.4 \%$ EM1 cells had $88 \%$ sensitivity and $67 \%$ specificity for hepatopathy).

These data show a strong association between exhausted phenotype of CD8 T cells, ILD and AIC, and strong association between CD8 T cell activation and non-infectious diarrhea. While the prevalence of CD127-PD- $1^{+} \mathrm{TIGIT}{ }^{+} \mathrm{CD} 28^{-}$exhausted cluster 8/12-like cells was a strong predictor of ILD, the proportion of $\mathrm{TIGIT}^{+}$cells also performed well in distinguishing such patients.

\section{Discussion}

In this study we explored the CD8 landscape of patients with CVID and healthy donors, with particular focus on the clinical heterogeneity of CVID as divided into infection only and complex disease groups.

We observed an increase of CD8 T cells in the peripheral blood of CVID patients, shifting from naïve towards more differentiated effector memory and TEMRA stages, in line with findings of earlier studies $[14,15]$. However, by combining a higher number of parameters in a single staining analysis we were able to show that $\mathrm{CD} 27^{+} 28^{-}$and $\mathrm{CD} 27^{-2} 28^{-}$effector memory T cells are particularly affected and differ in functional and phenotypic patterns from their $\mathrm{CD}^{2} 7^{+} 28^{+}$and $\mathrm{CD} 27^{-}$ $28^{+}$counterparts. Moreover, our work indicates that accumulation of several distinct populations, including functionally exhausted T cells, and activated T cells with immunoregulatory features, represent a major characteristic of CVID and is more pronounced in patients with complex disease.

Through unsupervised exploration of high-parametric dataset obtained with CyTOF we identified unique cellular clusters upregulated in CVID patients. Chief among these were cell clusters with high functional exhaustion scores, co-expression of inhibitory receptors including PD-1, TIGIT, 2B4, LAG-3, transcription factors Eomes, TOX, and intermediate expression of cytotoxic and proinflammatory molecules. These are all hallmarks of terminally differentiated exhausted cells $[29,39]$, which may be a product of an active feedback loop limiting damage in the context of recurrent stimulation with pathogen-derived antigenic stimuli $[6,7]$ or autoimmunity [40]. A CD127-PD- $1^{+} \mathrm{TIGIT}^{+} \mathrm{CD} 28^{-}$population corresponding to these exhausted clusters was detectable in the full cohort of 40 patients, upregulated in CVIDc and particularly strongly associated with ILD. The progenitors of exhausted cells which express CXCR5, intermediate levels of PD-1 and the transcription factor TCF1 [41,42] were preserved in our patients, enabling the maintenance of the terminally exhausted clusters. Patients in our study did not have overt clinical or laboratory signs of ongoing CMV infection, which has been previously shown to drive loss of CD127 and features of CD8 T cell senescence in patients with CVID $[17,43]$, and ELISpot assessment of CMV-specific T cells performed in a subset of our patients showed evidence of immune memory in only $2 / 7$ of those tested.

The expression of CXCR5 has also been described in recent years in regulatory CD8 T cells and follicular CD8 T cells, first in mice and later in humans [35,4446]. While the existence of regulatory CD8 T cells is still contentious in humans and better described in murine models [47], we've previously shown the existence of follicular CD8 T cells producing IL-10 in secondary lymphoid organs of patients with CVID and lymphadenopathy [48], which bore transcriptional and phenotypic markers of exhaustion. Here, we see a similar population of CD8 T cells in the peripheral blood, which express relatively high levels of CXCR5, high levels of PD-1, TIGIT, CTLA-4, lack the IL-7 receptor a subunit CD127, are strongly activated, proliferating and produce IL-10. Compared to other CD8 T cells, they also have a significantly higher average expression of FoxP3, which is the hallmark of regulatory CD4 T cells [49]. Staining for a corresponding CD127-PD-1 ${ }^{\text {high }}$ TIGIT $^{+}$CD28 $8^{+}$population in a larger cohort of patients revealed that these cells were not expanded in patients with non-infectious diarrhea and/or hepatopathy as it is seen in patients without these complications. As previously shown, $C D 21^{\text {low }} \mathrm{B}$ cells are associated with autoimmune phenomena [50] and it is thus feasible to speculate on the immunoregulatory role of PD- ${ }^{\text {high }}{ }^{\text {CXCR5 }}{ }^{+}$CD8 $\mathrm{T}$ cells in patients with complex form of CVID, similarly to the regulatory function of the $\mathrm{CD} 44^{+} \mathrm{CD} 122^{+} \mathrm{Ly} 49^{+} \mathrm{CD} 8 \mathrm{~T}$ cells shown in the murine model of autoimmune encephalomyelitis, which also upregulated the $\mathrm{CxCr} 5$ gene [36].

The diversity of clinical features seen in patients with CVIDc limited the robustness of changes we could note when stratifying cohorts purely based on the division between CVIDio and CVIDc. However, when stratifying patients based on individual complications, more robust patterns emerged. The loss of naïve T cell markers such as CD127 and upregulation of inhibitory receptors, in particular TIGIT, was very strongly associated with autoimmune cytopenia and interstitial lung disease in our cohort and showed significant sensitivity and specificity for history of these particular clinical features. Some data exists to suggest T-cell-mediated cytotoxicity as an alternative mechanism for platelet destruction in immune-mediated thrombocytopenia (ITP) [25] and both CD4 and CD8 T cells infiltrate the pulmonary interstitium of patients with CVID [51,52], but no causal link has yet been shown between CD8 T cell differentiation and ILD or AIC manifestation.

Interestingly, gastrointestinal complaints seen in our patients (non-infectious diarrhea, in some but not all cases accompanied by endoscopic features of enteropathy, with or without villous atrophy) were most strongly associated with CD8 T cell activation, rather than expansion of regulatory CD8 T cells or signs of exhaustion. CD8 T cell activation was previously noted in CVID as Viallard et al observed an inverse relation between naïve and activated CD8 T cells in 
CVID [22], Lanio et al. saw higher proportion of activated CD8 T cells in patients with splenomegaly [21], Carter et al. noted elevated proportion of HLA-DR ${ }^{+}$ cells but no association with autoimmune phenomena [19] and de Lollo noted higher proportion of CD127-38 ${ }^{+}$cells in patients with splenomegaly compared to healthy donors [20]. Non-infectious chronic diarrhea is a well-described complication of CVID associated with peripheral blood CD8 lymphocytosis, with the majority of intraepithelial lymphocytes seen in CVID enteropathy being CD8 ${ }^{+}$[23], further substantiating the association we observe in the peripheral blood. It's notable that Han et al. also found an expansion of activated CD38 ${ }^{+}$effector memory CD $8 \mathrm{~T}$ cells in the intestine of celiac disease patients challenged with gluten, with a corresponding aEß7 population in the peripheral blood, which were phenotypically similar to the "immunoregulatory" and highly activated cells we found [53], but which were not specifically associated with diarrhea in our cohort. Our own previous work failed to find association between peripheral a4ß7 T-cell numbers and gastrointestinal disease in CVID, but did not evaluate their activation or other phenotype [54]. While infection with norovirus may drive enteropathy in some antibody-deficient patients [55,56], all but 3 patients we tested were negative for norovirus PCR.

The accumulation of exhausted T cells in complicated CVID observed in this work fits to known mechanisms driving the differentiation of exhausted T cells, such as prolonged antigen stimulation in a chronic inflammatory milieu with inhibitory immune signaling. The specificity of these exhausted T cells in CVIDc, however, is unclear, such as whether they reflect dampened autoimmune responses or insufficient immunity against infectious pathogens, all of which could induce exhausted T cell phenotypes. This raises the question if targeting exhausted T cells could represent therapeutic strategies for symptomatic CVIDc patients. While the latter might potentially underly the expansion of activated T cells associated with infectious diarrhea and could profit from checkpoint therapy, in the context of autoimmune enteropathy and other autoimmune manifestations, such an approach is of high risk of boosting self-reactive T cells and resulting in enhanced autoimmunity. Thus, careful evaluation of the specificity of exhausted T cells in these scenarios are warranted in further studies.

\section{Conclusion}

In summary, in this study we show that CD8 T cells are highly differentiated, activated and show an expansion of effector memory populations with signs of increased T cell exhaustion in patients with CVID. These phenotypic changes strongly associate with particular clinical findings such as autoimmune cytopenia, interstitial lung disease and non-infectious diarrhea, prompting future prospective assessment of T cell phenotype in risk-stratifying patients with respect to screening of non-infectious complications before their clinical manifestation and supporting more aggressive treatment.

We also show a population of activated proliferating CD8 T cells which express CXCR5 and show phenotype and function congruent with their proposed immunoregulatory role. These cells were expanded in CVID patients without gastrointestinal manifestations and previously shown in secondary lymphoid organs of CVID patients. Their biology, ontology and functions should be explored in more detail, adding to their increasingly acknowledged role in chronic viral infections, autoimmunity, and cancer.

\section{Declarations}

\section{Acknowledgements}

Our thanks go out to the patients for their contribution to this study and to the multidisciplinary team of doctors, nurses and lab technicians who take care of them. We wish to thank Ina Harder, Sylvia Gutenberger, Bärbel Keller and Dominik Wieland for their help during the design and acquisition of data for this study. This study/project was supported by the FREEZE-Biobank, Center for Biobanking, Medical Center and Faculty of Medicine, University of Freiburg, Freiburg, Germany.

\section{Funding}

This study was funded by a grant issued by the Deutsche Forschungsgemeinschaft (grant no. SFB 1160 IMPATH, project A04 to K.W. and project A03 to B.B.), a personal grant of the European Society for Immunodeficiencies to A.K., and grants issued by the Czech health research council and Ministry of Health, Czech Republic (grant no. AZV NV18-05-00162 and grant no. NU20-05-00282).

\section{Conflict of Interest}

None of the authors has any potential financial conflict of interest related to this manuscript.

\section{Availability of data and material}

Data is available from authors upon reasonable request. Material is not available.

\section{Code availability}

Not applicable.

\section{Author's contributions}


AK designed the study, performed experiments, analysed data and wrote the manuscript. DF performed the experiments and provided clinical data. ES performed experiments. SU designed the study and reviewed the manuscript. ZP analysed the data. SG provided patient samples and clinical information. AS provided funding, interpreted the results and reviewed the manuscript. BB designed the experiments, interpreted results and co-wrote the manuscript. KW designed the study, provided patient samples, clinical information, interpreted results and co-wrote the manuscript.

\section{Ethics approval}

Written informed consent with participation in this study was obtained from all patients and control subjects in accordance with the ethical standards of the institutional and national research committee and with the 1964 Helsinki Declaration and its later amendments. The study was approved by local authorities (University of Freiburg Ethics Committee 239/1999 and 121/11).

\section{Consent to participate}

Written informed consent was obtained from all individual participants included in this study.

\section{Consent to publish}

No identifiable information is published as part of this study.

\section{References}

1. Bonilla FA, Barlan I, Chapel H, Costa-Carvalho BT, Cunningham-Rundles C, de la Morena MT, et al. International Consensus Document (ICON): Common Variable Immunodeficiency Disorders. J Allergy Clin Immunol Pract. 2016,4:38-59.

2. Cunningham-Rundles C. The many faces of common variable immunodeficiency. Hematology Am Soc Hematol Educ Program. NIH Public Access, 2012,301-5.

3. Wehr C, Kivioja T, Schmitt C, Ferry B, Witte T, Eren E, et al. The EUROclass trial: defining subgroups in common variable immunodeficiency. Blood. American Society of Hematology, 2008,111:77-85.

4. Giovannetti A, Pierdominici M, Mazzetta F, Marziali M, Renzi C, Mileo AM, et al. Unravelling the complexity of T cell abnormalities in common variable immunodeficiency. J Immunol. The American Association of Immunologists, 2007,178:3932-43.

5. Guazzi V, Aiuti F, Mezzaroma I, Mazzetta F, Andolfi G, Mortellaro A, et al. Assessment of thymic output in common variable immunodeficiency patients by evaluation of T cell receptor excision circles. Clin Exp Immunol. Wiley, 2002,129:346-53.

6. Le Coz C, Bengsch B, Khanna C, Trofa M, Ohtani T, Nolan BE, et al. Common variable immunodeficiency-associated endotoxemia promotes early commitment to the T follicular lineage. J Allergy Clin Immunol. Elsevier BV, 2019,144:1660-73.

7. Perreau M, Vigano S, Bellanger F, Pellaton C, Buss G, Comte D, et al. Exhaustion of bacteria-specific CD4 T cells and microbial translocation in common variable immunodeficiency disorders. J Exp Med. Rockefeller University Press, 2014,211:2033-45.

8. Unger S, Seidl M, van Schouwenburg P, Rakhmanov M, Bulashevska A, Frede N, et al. The TH1 phenotype of follicular helper T cells indicates an IFN- $\gamma-$ associated immune dysregulation in patients with CD21low common variable immunodeficiency. J Allergy Clin Immunol. Elsevier Inc., 2017,141:730-40.

9. Friedmann D, Unger S, Keller B, Rakhmanov M, Goldacker S, Zissel G, et al. Bronchoalveolar lavage fluid reflects a TH1-CD21low B-cell interaction in CVIDrelated interstitial lung disease. Front Immunol. Frontiers Media SA, 2020,11:616832.

10. Mannon PJ, Fuss IJ, Dill S, Friend J, Groden C, Hornung R, et al. Excess IL-12 but not IL-23 accompanies the inflammatory bowel disease associated with common variable immunodeficiency. Gastroenterology. Elsevier BV, 2006,131:748-56.

11. Berbers R-M, van der Wal MM, van Montfrans JM, Ellerbroek PM, Dalm VASH, van Hagen PM, et al. Chronically activated T-cells retain their inflammatory properties in common variable immunodeficiency. J Clin Immunol. Springer Science and Business Media LLC, 2021,41:1621-32.

12. Mouillot G, Carmagnat M, Gérard L, Garnier JL, Fieschi C, Vince N, et al. B-cell and T-cell phenotypes in CVID patients correlate with the clinical phenotype of the disease. J Clin Immunol. 2010,30:746-55.

13. Edwards ESJ, Bosco JJ, Aui PM, Stirling RG, Cameron PU, Chatelier J, et al. Predominantly Antibody-Deficient Patients With Non-infectious Complications Have Reduced Naive B, Treg, Th17, and Tfh17 Cells. Front Immunol. 2019,10:2593.

14. Viallard J-F, Ruiz C, Guillet M, Pellegrin J-L, Moreau J-F. Perturbations of the CD8(+) T-cell repertoire in CVID patients with complications. Results Immunol. Elsevier BV, 2013,3:122-8.

15. Bateman EAL, Ayers L, Sadler R, Lucas M, Roberts C, Woods A, et al. T cell phenotypes in patients with common variable immunodeficiency disorders: associations with clinical phenotypes in comparison with other groups with recurrent infections. Clin Exp Immunol. Wiley, 2012,170:202-11.

16. North ME, Webster AD, Farrant J. Primary defect in CD8+ lymphocytes in the antibody deficiency disease (common variable immunodeficiency): abnormalities in intracellular production of interferon-gamma (IFN-gamma) in CD28+ ('cytotoxic') and CD28- ('suppressor') CD8+ subsets. Clin Exp Immunol. Wiley, 1998,111:70-5.

17. Kuntz M, Goldacker S, Blum HE, Pircher H, Stampf S, Peter $\mathrm{H}-\mathrm{H}$, et al. Analysis of bulk and virus-specific CD8+ T cells reveals advanced differentiation of CD8+ T cells in patients with common variable immunodeficiency. Clin Immunol. Elsevier BV, 2011,141:177-86. 
18. Lollo C de, de Moraes Vasconcelos D, Oliveira LM da S, Domingues R, Carvalho GC de, Duarte AJ da S, et al. Chemokine, cytokine and type I interferon production induced by Toll-like receptor activation in common variable immune deficiency. Clin Immunol. Elsevier BV, 2016,169:121-7.

19. Carter CRD, Aravind G, Smalle NL, Cole JY, Savic S, Wood PMD. CVID patients with autoimmunity have elevated T cell expression of granzyme B and HLADR and reduced levels of Treg cells. J Clin Pathol. BMJ, 2013,66:146-50.

20. de Lollo C, de Moraes Vasconcelos D, da Silva Oliveira LM, de Oliveira Titz T, Carneiro-Sampaio M, Jacob CMA, et al. Impaired CD8+ T cell responses upon Toll-like receptor activation in common variable immunodeficiency. J Transl Med [Internet]. Springer Nature, 2016,14. Available from: http://dx.doi.org/10.1186/s12967-016-0900-2

21. Lanio N, Sarmiento E, Gallego A, Carbone J. Immunophenotypic profile of T cells in common variable immunodeficiency: is there an association with different clinical findings? Allergol Immunopathol (Madr). Codon Publications, 2009,37:14-20.

22. Viallard J-F, Blanco P, André M, Etienne G, Liferman F, Neau D, et al. CD8+HLA-DR+ T lymphocytes are increased in common variable immunodeficiency patients with impaired memory B-cell differentiation. Clin Immunol. Elsevier BV, 2006,119:51-8.

23. Malamut G, Verkarre V, Suarez F, Viallard J-F, Lascaux A-S, Cosnes J, et al. The enteropathy associated with common variable immunodeficiency: the delineated frontiers with celiac disease. Am J Gastroenterol. Ovid Technologies (Wolters Kluwer Health), 2010,105:2262-75.

24. Szablewski V, René C, Costes V. Indolent cytotoxic T cell lymphoproliferation associated with nodular regenerative hyperplasia: a common liver lesion in the context of common variable immunodeficiency disorder. Virchows Arch. Springer Science and Business Media LLC, 2015,467:733-40.

25. Olsson B, Andersson P-O, Jernås M, Jacobsson S, Carlsson B, Carlsson LMS, et al. T-cell-mediated cytotoxicity toward platelets in chronic idiopathic thrombocytopenic purpura. Nat Med. Springer Science and Business Media LLC, 2003,9:1123-4.

26. McKinney EF, Lee JC, Jayne DRW, Lyons PA, Smith KGC. T-cell exhaustion, co-stimulation and clinical outcome in autoimmunity and infection. Nature. Springer Science and Business Media LLC, 2015,523:612-6.

27. McLane LM, Abdel-Hakeem MS, Wherry EJ. CD8 T cell exhaustion during chronic viral infection and cancer. Annu Rev Immunol. Annual Reviews, 2019,37:457-95.

28. Bengsch B, Ohtani T, Khan O, Setty M, Manne S, O’Brien S, et al. Epigenomic-guided mass cytometry profiling reveals disease-specific features of exhausted CD8 T cells. Immunity. Elsevier BV, 2018,48:1029-1045.e5.

29. Winkler F, Bengsch B. Use of mass cytometry to profile human T cell exhaustion. Front Immunol. Frontiers Media SA, 2019,10:3039.

30. Bendall SC, Davis KL, Amir E-AD, Tadmor MD, Simonds EF, Chen TJ, et al. Single-cell trajectory detection uncovers progression and regulatory coordination in human B cell development. Cell. 2014,157:714-25.

31. Van Gassen S, Callebaut B, Van Helden MJ, Lambrecht BN, Demeester P, Dhaene T, et al. FlowSOM: Using self-organizing maps for visualization and interpretation of cytometry data. Cytometry A. Wiley, 2015,87:636-45.

32. Mclnnes L, Healy J, Melville J. UMAP: Uniform Manifold Approximation and Projection for Dimension Reduction [Internet]. arXiv [stat.ML]. 2018. Available from: http://arxiv.org/abs/1802.03426

33. Roederer M, Nozzi JL, Nason MC. SPICE: Exploration and analysis of post-cytometric complex multivariate datasets. Cytometry A. Wiley, 2011,79A:16774.

34. Brenchley JM, Karandikar NJ, Betts MR, Ambrozak DR, Hill BJ, Crotty LE, et al. Expression of CD57 defines replicative senescence and antigen-induced apoptotic death of CD8+ T cells. Blood. American Society of Hematology, 2003,101:2711-20.

35. Fousteri G, Kuka M. The elusive identity of CXCR5+ CD8 T cells in viral infection and autoimmunity: Cytotoxic, regulatory, or helper cells? Mol Immunol. Elsevier BV, 2020,119:101-5.

36. Saligrama N, Zhao F, Sikora MJ, Serratelli WS, Fernandes RA, Louis DM, et al. Opposing T cell responses in experimental autoimmune encephalomyelitis. Nature. Springer Science and Business Media LLC, 2019,572:481-7.

37. Abdel-Hakeem MS, Manne S, Beltra J-C, Stelekati E, Chen Z, Nzingha K, et al. Epigenetic scarring of exhausted T cells hinders memory differentiation upon eliminating chronic antigenic stimulation. Nat Immunol. Springer Science and Business Media LLC, 2021,22:1008-19.

38. Ando M, Ito M, Srirat T, Kondo T, Yoshimura A. Memory T cell, exhaustion, and tumor immunity. Immunol med. Informa UK Limited, 2020,43:1-9.

39. Wherry EJ, Kurachi M. Molecular and cellular insights into T cell exhaustion. Nat Rev Immunol. Springer Science and Business Media LLC, 2015,15:48699.

40. Pawelec G. Is there a positive side to T cell exhaustion? Front Immunol. Frontiers Media SA, 2019,10:111.

41. Chu T, Berner J, Zehn D. Two parallel worlds of memory T cells. Nat. Immunol. Springer Science and Business Media LLC, 2020. p. 1484-5.

42. Utzschneider DT, Charmoy M, Chennupati V, Pousse L, Ferreira DP, Calderon-Copete S, et al. T cell factor 1-expressing memory-like CD8+ T cells sustain the immune response to chronic viral infections. Immunity. Elsevier BV, 2016,45:415-27.

43. Marashi SM, Raeiszadeh M, Enright V, Tahami F, Workman S, Chee R, et al. Influence of cytomegalovirus infection on immune cell phenotypes in patients with common variable immunodeficiency. J Allergy Clin Immunol. Elsevier BV, 2012,129:1349-1356.e3.

44. Im SJ, Hashimoto M, Gerner MY, Lee J, Kissick HT, Burger MC, et al. Defining CD8+ T cells that provide the proliferative burst after PD-1 therapy. Nature. Springer Science and Business Media LLC, 2016,537:417-21.

45. Quigley MF, Gonzalez VD, Granath A, Andersson J, Sandberg JK. CXCR5+ CCR7- CD8 T cells are early effector memory cells that infiltrate tonsil B cell follicles. Eur J Immunol. Wiley, 2007,37:3352-62.

46. Kim H-J, Verbinnen B, Tang X, Lu L, Cantor H. Inhibition of follicular T-helper cells by CD8(+) regulatory T cells is essential for self tolerance. Nature. Springer Science and Business Media LLC, 2010,467:328-32.

Page $9 / 13$ 
47. Smith TRF, Kumar V. Revival of CD8+ Treg-mediated suppression. Trends Immunol. Elsevier BV, 2008,29:337-42.

48. Klocperk A, Unger S, Friedmann D, Seidl M, Zoldan K, Pfeiffer J, et al. Exhausted phenotype of follicular CD8 T cells in CVID. J Allergy Clin Immunol. Elsevier BV, 2020,146:912-915.e13.

49. Tang Q, Bluestone JA. The Foxp3+ regulatory T cell: a jack of all trades, master of regulation. Nat Immunol. Springer Nature, 2008,9:239-44.

50. Wehr C, Eibel H, Masilamani M, Illges H, Schlesier M, Peter H-H, et al. A new CD21low B cell population in the peripheral blood of patients with SLE. Clin Immunol. Elsevier BV, 2004,113:161-71.

51. Dhalla F, Lochlainn DJM, Chapel H, Patel SY. Histology of interstitial lung disease in common variable immune deficiency. Front Immunol. Frontiers Media SA, 2020,11:605187.

52. Patel S, Anzilotti C, Lucas M, Moore N, Chapel H. Interstitial lung disease in patients with common variable immunodeficiency disorders: several different pathologies? Clin Exp Immunol. Wiley, 2019,198:212-23.

53. Han A, Newell EW, Glanville J, Fernandez-Becker N, Khosla C, Chien Y-H, et al. Dietary gluten triggers concomitant activation of CD4+ and CD8+ a $\mathrm{T}$ cells and $\gamma \delta$ T cells in celiac disease. Proc Natl Acad Sci U S A. Proceedings of the National Academy of Sciences, 2013,110:13073-8.

54. de Melo KM, Unger S, Keller B, Gutenberger S, Stumpf I, Goldacker S, et al. Increase of circulating a4ß7 conventional memory CD4 and regulatory T cells in patients with common variable immunodeficiency (CVID). Clin Immunol. 2017,180:80-3.

55. van de Ven AAJM, Janssen WJM, Schulz LS, van Loon AM, Voorkamp K, Sanders EAM, et al. Increased prevalence of gastrointestinal viruses and diminished secretory immunoglobulin a levels in antibody deficiencies. J Clin Immunol. Springer Science and Business Media LLC, 2014,34:962-70.

56. Woodward J, Gkrania-Klotsas E, Kumararatne D. Chronic norovirus infection and common variable immunodeficiency. Clin Exp Immunol. Wiley, 2017,188:363-70.

\section{Tables}

Table 1 Cohort description. $\mathrm{AIC}=$ autoimmune cytopenia, CVIDC $=$ complex CVID, CVIDio $=$ infection only CVID, ILD = interstitial lung disease, $\mathrm{LAD}=$ Iymphadenopathy, I.b. = likely benign, NA = not available, no IS = no immunosuppression, RTX = rituximab, Unknown = whole exome or panel sequencing performed, but no hits explaining phenotype were found, VUS = variant of unknown significance 


\begin{tabular}{|c|c|c|c|c|c|c|c|c|c|c|c|c|}
\hline ID & Category & $\begin{array}{l}\text { Genetic } \\
\text { cause }\end{array}$ & $\begin{array}{l}\text { Freiburg } \\
\text { classification }\end{array}$ & $\begin{array}{l}\text { EUROclass } \\
\text { classification }\end{array}$ & Sex & Splenomegaly & LAD & Lymphoma & AIC & Diarrhea & Norovirus & IL \\
\hline 1 & CVIDc & CTLA-4 & la & B+smB+21lowTrnorm & $\mathrm{F}$ & 1 & 1 & 1 & 1 & 1 & 0 & 1 \\
\hline 2 & CVIDc & NFkB1 & $\mathrm{lb}$ & B+smB-21lowTrnorm & $\mathrm{F}$ & 1 & 1 & 0 & 1 & 1 & 0 & 1 \\
\hline 3 & CVIDc & Unknown & la & B+smB-21lowTrhigh & $\mathrm{F}$ & 1 & 1 & 0 & 0 & 0 & NA & 1 \\
\hline 4 & CVIDc & NA & $\mathrm{lb}$ & B+smB-21normTrnorm & $\mathrm{F}$ & 1 & 0 & 0 & 1 & 0 & NA & 0 \\
\hline 5 & CVIDc & NA & $\mathrm{lb}$ & B+smB-21lowTrnorm & M & 1 & 0 & 0 & 0 & 0 & NA & 1 \\
\hline 6 & CVIDc & Unknown & la & B+smB-21lowTrnorm & M & 1 & 0 & 0 & 0 & 1 & 1 & 0 \\
\hline 7 & CVIDc & Unknown & $\mathrm{lb}$ & B+smB-21lowTrnorm & $\mathrm{F}$ & 1 & 1 & 0 & 0 & 0 & NA & 1 \\
\hline 8 & CVIDc & Unknown & la & B+smB-21lowTrhigh & M & 1 & 1 & 0 & 1 & 1 & 0 & 0 \\
\hline 9 & CVIDc & Unknown & $\mathrm{lb}$ & B+smB-21lowTrhigh & $\mathrm{F}$ & 1 & 1 & 0 & 1 & 1 & 0 & 1 \\
\hline 10 & CVIDc & Unknown & la & B+smB-21lowTrnorm & $\mathrm{F}$ & 1 & 1 & 0 & 1 & 1 & 0 & 1 \\
\hline 11 & CVIDc & CTLA-4 & la & B+smB-21lowTrnorm & M & 1 & 0 & 1 & 1 & 1 & 0 & 0 \\
\hline 12 & CVIDc & NFkB2 & $\mathrm{lb}$ & B+smB-21normTrhigh & $\mathrm{F}$ & 0 & 0 & 0 & 0 & 0 & NA & 0 \\
\hline 13 & CVIDc & $\begin{array}{l}\text { NFkB1 + } \\
\text { TACI I.b. }\end{array}$ & la & B+smB+21lowTrnorm & M & 0 & 0 & 0 & 0 & 1 & NA & 0 \\
\hline 14 & CVIDc & Unknown & $\mathrm{lb}$ & B+smB-21normTrnorm & $\mathrm{F}$ & 1 & 0 & 0 & 0 & 0 & NA & 0 \\
\hline 15 & CVIDc & Unknown & la & B+smB-21lowTrnorm & $\mathrm{F}$ & 1 & 1 & 0 & 0 & 1 & 0 & 1 \\
\hline 16 & CVIDc & Fas & $\mathrm{lb}$ & B+smB-21normTrhigh & $\mathrm{F}$ & 1 & 0 & 0 & 0 & 1 & 0 & 0 \\
\hline 17 & CVIDc & NA & $\mathrm{lb}$ & B+smB-21lowTrnorm & $\mathrm{F}$ & 1 & 0 & 0 & 0 & 1 & NA & 1 \\
\hline 18 & CVIDc & TACI I.b. & la & B+smB-21lowTrnorm & M & 1 & 1 & 0 & 1 & 1 & NA & 1 \\
\hline 19 & CVIDc & Unknown & $\mathrm{lb}$ & B+smB-21normTrnorm & M & 1 & 0 & 0 & 0 & 1 & 0 & 1 \\
\hline 20 & CVIDc & Unknown & ॥ & B+smB+21lowTrnorm & $\mathrm{F}$ & 1 & 1 & 0 & 1 & 1 & 0 & 1 \\
\hline 21 & CVIDc & Unknown & la & B+smB-21lowTrnorm & $\mathrm{F}$ & 1 & 1 & 0 & 0 & 1 & 0 & 1 \\
\hline 22 & CVIDc & Unknown & la & B+smB+21lowTrnorm & M & 1 & 1 & 0 & 1 & 0 & NA & 1 \\
\hline 23 & CVIDc & Unknown & la & B+smB+21lowTrnorm & M & 1 & 1 & 0 & 1 & 0 & NA & 0 \\
\hline 24 & CVIDc & Unknown & la & B+smB-21lowTrhigh & $\mathrm{F}$ & 1 & 1 & 0 & 1 & 1 & 1 & 1 \\
\hline 25 & CVIDc & Unknown & II & $\mathrm{B}+\mathrm{smB}+21$ normTrnorm & $\mathrm{F}$ & 1 & 0 & 0 & 0 & 1 & 0 & 1 \\
\hline 26 & CVIDc & $\begin{array}{l}\text { VUS in } \\
\text { SOCS1 }\end{array}$ & la & $\begin{array}{l}\text { B-, previously B+smB- } \\
21 \text { low }\end{array}$ & $\mathrm{F}$ & 1 & 0 & 0 & 1 & 1 & NA & 0 \\
\hline 27 & CVIDc & Unknown & $\mathrm{lb}$ & B+smB-21lowTrnorm & M & 1 & 1 & 0 & 0 & 1 & 0 & 0 \\
\hline 28 & CVIDc & NFkB1 & $\mathrm{lb}$ & B+smB-21normTrnorm & M & 0 & 1 & 0 & 0 & 1 & 1 & 0 \\
\hline 29 & CVIDc & LRBA & la & B- & M & 1 & 0 & 0 & 1 & 1 & 0 & 1 \\
\hline 30 & CVIDc & Unknown & ॥ & $\mathrm{B}+\mathrm{smB}+21$ normTrnorm & M & 1 & 0 & 0 & 0 & 0 & NA & 0 \\
\hline 31 & CVIDio & Unknown & $\mathrm{lb}$ & B+smB-21normTrnorm & M & 1 & 0 & 0 & 0 & 0 & NA & 0 \\
\hline 32 & CVIDio & Unknown & $\mathrm{lb}$ & B+smB-21normTrnorm & $\mathrm{F}$ & 0 & 0 & 0 & 0 & 0 & NA & 0 \\
\hline 33 & CVIDio & NA & la & $\mathrm{B}+\mathrm{smB}+21$ normTrhigh & M & 0 & 0 & 0 & 0 & 0 & NA & 0 \\
\hline 34 & CVIDio & NFkB1 & la & B+smB-21lowTrnorm & M & 0 & 0 & 0 & 0 & 0 & NA & 0 \\
\hline 35 & CVIDio & Unknown & la & B- & M & 1 & 0 & 0 & 0 & 0 & NA & 0 \\
\hline 36 & CVIDio & BAFFR & $\mathrm{lb}$ & $\mathrm{B}+\mathrm{smB}+21$ normTrhigh & M & 0 & 0 & 0 & 0 & 0 & 0 & 0 \\
\hline 37 & CVIDio & Unknown & $\mathrm{lb}$ & B+smB-21normTrhigh & M & 1 & 0 & 0 & 0 & 0 & NA & 0 \\
\hline
\end{tabular}




\begin{tabular}{|lllllllllll}
38 & CVIDio & Unknown & Ib & B+smB+21normTrnorm & M & 0 & 0 & 0 & 0 & 0 \\
39 & CVIDio & Unknown & Ib & B+smB-21normTrnorm & F & 1 & 0 & 0 & 0 & 0 \\
\hline 40 & CVIDio & Unknown & Ib & B+smB-21normTrnorm & F & 0 & 0 & 0 & 0 & 0 \\
\hline
\end{tabular}

\section{Figures}

\section{Figure 1}

Clinical features of the cohort and changes in CD8 differentiation stages. A, complications seen in the studied cohort of CVID patients ( $n=40,11$ CVIDio and 29 CVIDc). B, EURO classification of the studied cohort. C, proportion of CD8 T cells in HD, CVIDio and CVIDc. D, E, differentiation stages of CD8 T cells assessed by flow cytometry. F, distribution of differentiation stages in Wanderlust-derived pseudotime on 12 samples (4 HD, 4 CVIDio, 4 CVIDc) assessed by CyTOF. G, changes in basic differentiation markers over Wanderlust-derived pseudotime. H, heatmap showing phenotype of PBMCs from 4 HD, 4 CVIDio and 4 CVIDc after stimulation with PMA and ionomycin, assessed by CyTOF. Expression normalized per marker, color scale corresponds to Z-values

\section{Figure 2}

Multiparametric analysis of the CD8 compartment as assessed by CyTOF ( $n=12,4$ HD, 4 CVIDio and 4 CVIDc). A, UMAP visualization of CD8 T cells in concatenated HD, CVIDio and CVIDc cohorts. B, FlowSOM clusters displayed in UMAP dimensions. C, FlowSOM cluster proportions in the three cohorts. D, FlowSOM cluster phenotype heatmap, with calculated FES. E, cluster distribution in Wanderlust pseudotime as corresponds with canonical naïve, CM, EM and TEMRA populations, with clusters significantly affected in CVID marked with an asterisk

\section{Figure 3}

Flow cytometric evaluation of single markers and CyTOF-like clusters in a larger cohort ( $n=57,17$ HD, 11 CVIDio and 29 CVIDc). A, heatmap showing expression levels of single markers on bulk CD8 T cells in HD, CVIDio and CVIDc $(n=57)$. B, activation, C, proliferation, $D$, cytotoxicity and E, differentiation and exhaustion markers on bulk CD8 T cells in HD, CVIDio and CVIDc. F, prevalence of CD127-PD-1+TIGIT+CD28- (Cluster 8/12-like) and CD127-PD-

$1^{\text {high }} \mathrm{TIGIT}^{+}$CD28 $8^{+}$(Cluster 16-like) cells assessed by flow cytometry $(\mathrm{n}=32)$ in HD, CVIDio and CVIDc. G, correlation between Cluster 8/12-like and Cluster 16like populations assessed by flow cytometry and Cluster $8+12$ and Cluster 16 cells assessed by CyTOF in identical samples $(n=12)$. $H$, heatmap showing expression of 39 markers assessed by CyTOF in Cluster 8, 12, 16, Cluster 8/12-like, Cluster 16-like and Naïve $\left(C D 45 R A^{+} C C R 7^{+}\right)$cells $(n=12,4$ HD, 4 CVIDio and 4 CVIDc). I, co-localization of Clusters 8, 12, 16 and Cluster 8/12-like and Cluster 16-like cells assessed by CyTOF in UMAP dimensionality reduction space ( $\mathrm{n}=$ $12,4$ HD, 4 CVIDio and 4 CVIDc). J, K, correlation of CD8 phenotype assessed by flow cytometry with the EURO classification ( $n=57)$. 


\section{A}

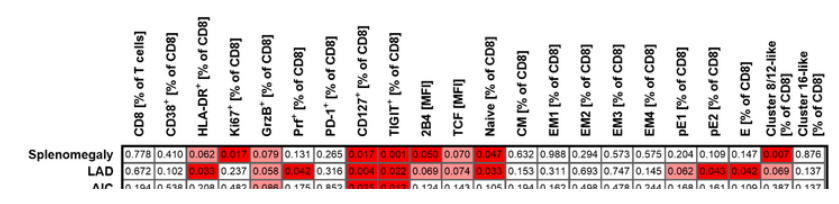

\section{Figure 4}

Clinical feature prediction in large cohort of CVID patients assessed by flow cytometry ( $n=57,17$ HD, 11 CVIDio and 29 CVIDc). A, p-values of main CD8 features as differentiating factors for individual clinical complications. B, cluster 8/12-like, CD127+ and TIGIT ${ }^{+}$CD8 T cells in patients with/without ILD. C, $\mathrm{CD}_{127^{+}}$and $\mathrm{TIGIT}^{+} \mathrm{CD} 8 \mathrm{~T}$ cells in patients with/without AIC. D, cluster 16-like, CD38 ${ }^{+}$and HLA-DR ${ }^{+}$CD8 T cells in patients with/without diarrhea. E, cluster $16-$ like, EM1 and bulk CD8 T cells in patients with/without hepatopathy. F, receiver-operating (ROC) curves for cluster 8/12-like, cluster 16-like, CD127 ${ }^{+}, \mathrm{TIGIT}^{+}$, $\mathrm{CD}_{38}{ }^{+}, \mathrm{HLA}-\mathrm{DR}^{+}$and bulk CD8 T cells and the presence/absence of ILD, AIC, diarrhea and hepatopathy.

\section{Supplementary Files}

This is a list of supplementary files associated with this preprint. Click to download.

- SupplementalMaterials20220411JOCl.docx 